\title{
Vergleich der nationalen Anhänge der EN 1992-1-1 zum Thema Querkraft und Durchstanzen
}

Der Eurocode 2 (EN 1992-1-1) beschäftigt sich mit der Bemessung und Konstruktion von Stahlbeton- und Spannbetontragwerken. Eine eingehende Betrachtung und der Vergleich der nationalen Anhänge sind notwendig, um die Unterschiede der national festgelegten Berechnungsparameter zu verifizieren. Dieser Aufsatz gibt einen Überblick über die gegenwärtige Situation und zeigt die wesentlichen Unterschiede der nationalen Anhänge der EN 1992-1-1 zu den Themen Querkraft und Durchstanzen auf.
Shear and Punching Shear verifications in comparison to the national annexes of the Eurocode 2

In order to get the Eurocodes accepted, it was necessary to allow national choices with regard to parameters where no agreement between the countries involved could be reached. For the part EN 1992-1-1 “Design of concrete structures Part I: General rules and rules for buildings" altogether 121 parameters have been defined which are subject to national choices. A comparison of the national choices can show where in future easily agreement can be reached. On the other hand parameters can be identified for which significant disagreement exist, which invites to further discussions and even research. In this paper, as an experiment, the nationally defined parameters of 18 countries are compared for the chapters on shear and punching, which subjects are now, for several reasons, again in the centre of interest.

verglichen wurden. Diese Staaten sind Österreich (AT), Belgien (BE), Zypern (CY), Dänemark (DK), Frankreich (FR), Vereinigtes Königreich (UK), Deutschland (DE), Griechenland (GR), Ungarn (HU), Italien (IT), die Niederlande (NL), Norwegen (NO), Polen (PL), Portugal (PT), Slowakei (SK), Spanien (ES), Finnland (FI) und Schweden (SE).

Die Kapitel 6.2.1 bis 6.2.3 und 6.4 der EN 1992-1-1 beinhalten 14 nationale Festlegungen in acht Absätzen, z. B. $\mathrm{C}_{\mathrm{Rd}, \mathrm{c}}, \mathrm{v}_{\min }$ und $\mathrm{k}_{1}$ (im Kapitel 6.2 .2 (1) und 6.4.4 (1)), $\theta$ (Kapitel 6.2.3 (2)), v (Kapitel 6.2.2 (6)), $v_{1}$ (Kapitel 6.2.3 (3)), $\alpha_{c w}$ (Kapitel 6.2.3 (3)), $\beta$ (Kapitel 6.4.3 (6)), $v_{\text {Rd,max }}$ (Kapitel 6.4.5 (3)), k (Kapitel 6.2.4 (6) und 6.4.5 (4)) und $\gamma_{\mathrm{c}}\left(\right.$ im Kapitel 2.4.2.4(1), $\mathrm{C}_{\mathrm{Rd}, \mathrm{c}}$ ).

Bild 1 zeigt die Anzahl der Abweichungen nationaler Parameter von den empfohlenen Werten des Eurocode 2 Dokuments. Der deutsche nationale Anhang ist jener mit den meisten Unterschieden im Vergleich zu den anderen betrachteten Ländern. Spanien, Dänemark, Frankreich, Finnland und Italien sind jene Länder, welche mit 4 bis 5 Unterschieden in den nationalen Werten folgen. Der einzige national bestimmte Parameter, der in allen untersuchten nationalen Anhängen nicht vom empfohlenen Wert abweicht, ist der Faktor k aus dem Kapitel 6.4, mit dem festgelegt wird, auf welcher maximalen Distanz die Durchstanzbewehrung vom äußeren kritischen Rundschnitt entfernt sein darf. Der Bemessungswert des maximalen Durchstanzwiderstands $\mathrm{V}_{\mathrm{Rd} \text {,max }}$, die minimale Querkrafttragfähigkeit $v_{\text {min }}$ bei Bauteilen ohne Querkraftbewehrung, der Druckstrebenwinkel $\theta$ und der maximal 
betreffend Kapitel 6.2.1 bis 6.2.3 und 6.4 EN 1992-1-1

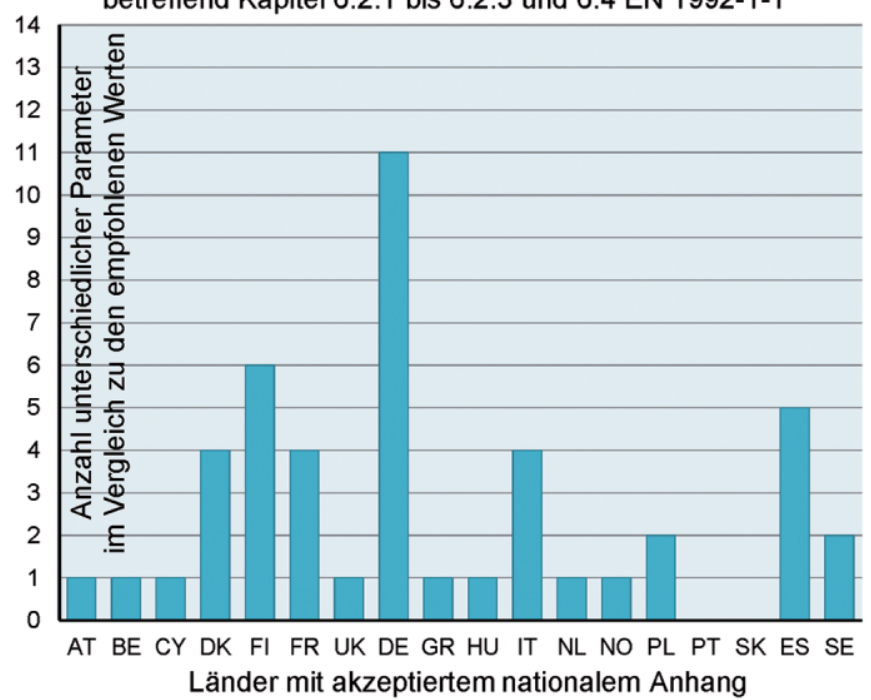

Bild 1 Länder und Anzahl der von den empfohlenen Werten des EN 1992-1-1 (Kapitel 6.2.1 bis 6.2.3 und 6.4) abweichenden nationalen Parameter Countries and the number of nationally defined parameters for which deviating choices have been made (EN 1992-1-1, chapters 6.2.1 to 6.2.3 and 6.4)

zulässige Querkraftwiderstand $V_{R d, \max }$ sind die Werte mit den größten Unterschieden in dieser Untersuchung.

\section{Querkrafttragfähigkeit 2.1 Vorbemerkungen}

Laut 6.2.2 der EN 1992-1-1 gilt für den Bemessungswert der Schubtragfähigkeit von Bauteilen ohne Querkraftbewehrung die folgende Formulierung:

$$
V_{R d, c}=\left[C_{R d, c} k\left(100 \rho_{l} f_{c k}\right)^{1 / 3}+\kappa_{1} \sigma_{c p}\right] b_{w} d
$$

mit einem Minimum von

$$
V_{R d, c}=\left(v_{\min }+k_{1} \sigma_{c p}\right) b_{w} d
$$

Für die Bemessungswerte der Querkrafttragfähigkeit von Bauteilen mit Querkraftbewehrung gilt

$$
V_{R d, s}=\frac{A_{s w}}{s} z f_{y w d} \cot \theta
$$

mit einem Maximum von

$$
V_{R d, \text { max }}=\alpha_{c w} b_{w} z v_{1} f_{c d} \frac{1}{(\cot \theta+\tan \theta)}
$$

\subsection{Querkrafttragfähigkeit ohne Querkraftbewehrung}

Die Gl. (1) basiert auf einer statistischen Analyse von 176 Versuchsergebnissen von KÖNIG und FIScHER [1]. Diese Auswahl wurde mit dem Ziel einer möglichst gleichmäßigen Verteilung der wichtigsten Parameter wie Betondruckfestigkeit $f_{c}$, Bewehrungsgrad in Längsrichtung $\rho_{1}$

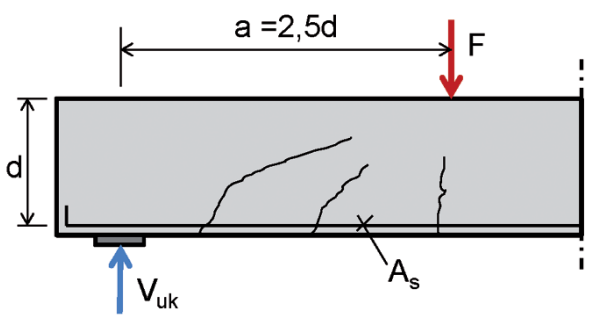

Bild 2 Ungünstigste Position einer Einzellast in der Nähe vom Auflager im Querkraftversuch, aus [2]

Most unfavourable position of load near to support to determine $v_{\min }[2]$

und statische Nutzhöhe d (unter Berücksichtigung des Maßstabsfaktors k) getroffen. KÖNIG und FIsCHER bezogen die Herleitung der Bemessungsformel nicht auf die $5 \%$-Quantile, gingen aber von einem Zuverlässigkeitsbeiwert $\beta=3,8$ aus, der nach EN1990 für neue Konstruktionen der Klasse 2 entspricht. Diese Betrachtung führt zu dem Beiwert $C_{R d, c}=0,12$. Ein Nachteil dieser Formulierung ist, dass das Sicherheitsniveau in Gl. (1) nicht sichtbar ist. Diese Überlegung hat dazu geführt, dass der Wert $\mathrm{C}_{\mathrm{Rd}, \mathrm{c}}=0,12$ durch den Ausdruck $\left(0,18 / \gamma_{\mathrm{c}}\right)$ ersetzt wurde. Der Normalfall mit $\gamma_{c}=1,5$ führt $\mathrm{zu} \mathrm{C}_{\mathrm{Rd}, \mathrm{c}}=0,12$. Dieser Wert wurde als national festzulegender Parameter deklariert, mit $\mathrm{C}_{\mathrm{Rd}, \mathrm{c}}=\left(0,18 / \gamma_{\mathrm{c}}\right)$ als empfohlenem Wert. Von allen Ländern dieser Untersuchung weichen nur Norwegen und Deutschland von diesem empfohlenen Wert ab. Norwegen knüpft an den Wert $C_{R d, c}=\left(0,18 / \gamma_{c}\right)$ die Bedingung, dass der Größtkorndurchmesser der Gesteinskörnung mindestens $16 \mathrm{~mm}$ beträgt, und weiterhin, dass wenigstens $50 \%$ des Gesamtzuschlags aus grobem Korn bestehen. Zusätzlich sollen Kalkstein und andere Zuschläge mit niedriger Festigkeit vermieden werden. Wenn eine der genannten Bedingungen nicht eingehalten wird, so gilt $C_{R d, c}=\left(0,15 / \gamma_{c}\right)$. Weiterhin gilt die Einschränkung, dass für $\mathrm{f}_{\mathrm{ck}}$ in den Gln. (1) und (2) kein größerer Wert als $65 \mathrm{MPa}$ eingeführt werden darf. Diese Überlegungen beabsichtigen sicherzustellen, dass eine ausreichende Rissverzahnung vorhanden ist. Deutschland geht grundsätzlich von $\left(0,15 / \gamma_{c}\right)$ aus.

Die Gl. (2) liefert ein Minimum für die Querkrafttragfähigkeit von Bauteilen ohne Querkraftbewehrung. Der Grund, weshalb dieser Term $v_{\min }$, zusätzlich zu Gl. (1), erforderlich ist, liegt im Grunde genommen darin, dass Gl. (1) eine Funktion des Bewehrungsgehalts $\rho_{1}$ ist. Für kleine Werte von $\rho_{1}$ ist Querkraftversagen höchst unwahrscheinlich, weil Biegeversagen maßgebend ist. Deshalb wurde für die Querkrafttragfähigkeit die untere Grenze $v_{\min }$ abgeleitet, basierend auf dem in Bild 2 vorgestellten Lastfall. Es wird angenommen, dass der ungünstigste Fall in Bezug auf Schubversagen eintritt, wenn eine Einzellast im Abstand 2,5d vom Auflager aufgebracht wird.

Dann kann berechnet werden, für welchen Wert $\rho_{1}$ Fließen der Längsbewehrung über Querkraftversagen maßgebend wird. Es wurde bei dieser Herleitung von einer $5 \%$ Untergrenze für die Querkrafttragfähigkeit von 


$$
V_{u k}=0,15 k\left(100 \rho_{l} f_{c k}\right)^{1 / 3}
$$

ausgegangen. Für die Biegetragfähigkeit wurde vereinfachend

$$
M_{u k}=0,9 d\left(\rho_{l} b d\right) f_{y k}
$$

angesetzt.

Durch Gleichsetzen der Gln. (5) und (6) findet man für den Bewehrungsgehalt, wobei der Übergang von Querkraftversagen zu Biegeversagen auftritt, den Ausdruck

$$
\rho_{l}=\frac{2,68 k^{3 / 2} f_{c k}^{1 / 2}}{f_{y k}^{3 / 2}}
$$

Führt man diesen Wert in Gl. (1) mit $\mathrm{C}_{\mathrm{Rd}, \mathrm{c}}=0,12$ ein, so findet man

$$
v_{\min }=\frac{0,78 k^{3 / 2} f_{c k}^{1 / 2}}{f_{y k}^{1 / 2}}
$$

Aus praktischen Gründen wurde davon ausgegangen, dass für Neubauten im Regelfall Betonstahl mit $\mathrm{f}_{y k}=$ $500 \mathrm{MPa}$ verwendet wird. Führt man diesen Wert in Gl. (8) ein, so findet man den in EN 1992-1-1, Kap. 6.2.2, für die untere Grenze der Querkrafttragfähigkeit vorgeschlagenen Ausdruck

$$
v_{\min }=0,035 k^{3 / 2} f_{c k}^{1 / 2}
$$

Die Länder dürfen jedoch von diesem Wert abweichen. Betreffend der Definition von $v_{\min }$ haben 13 von den 18 Ländern entschieden, den empfohlenen Wert aus Gl. (9) zu übernehmen. Einige wenige bevorzugen eine Harmonisierung von Gl. (9) mit den Gln. (1) und (2), in dem der Faktor $\gamma_{c}$ eingeführt wird, wodurch Gl. (9) ersetzt wird durch

$$
v_{\min }=\frac{0,053}{\gamma_{c}} k^{3 / 2} f_{c k}^{1 / 2}
$$

was für $\gamma_{c}=1,5$ dieselben Werte wie Gl. (9) ergibt. Die übrigen drei Länder (Deutschland, Spanien und Frankreich) weichen sehr beträchtlich von den empfohlenen Werten ab (Bild 3).

Deutschland definiert eine konservativere Formulierung für Querschnittshöhen über $600 \mathrm{~mm}$. Für d $>800 \mathrm{~mm}$ soll der Beiwert 0,053 in Gl. (10) durch 0,0375 ersetzt werden, was eine Reduktion mit einem Faktor 0,71 bedeutet. Zwischen 600 und $800 \mathrm{~mm}$ ist Interpolation erlaubt. Den Hintergrund hierzu findet man in [3]

Spanien ersetzt Gl. (9) durch

$$
v_{\min }=\frac{0,075}{\gamma_{c}} k^{3 / 2} f_{c k}^{1 / 2}
$$

Diese Gleichung ergibt Werte, welche um $41 \%$ höher als die empfohlenen sind. Belgien erlaubt einen Erhöhungs-

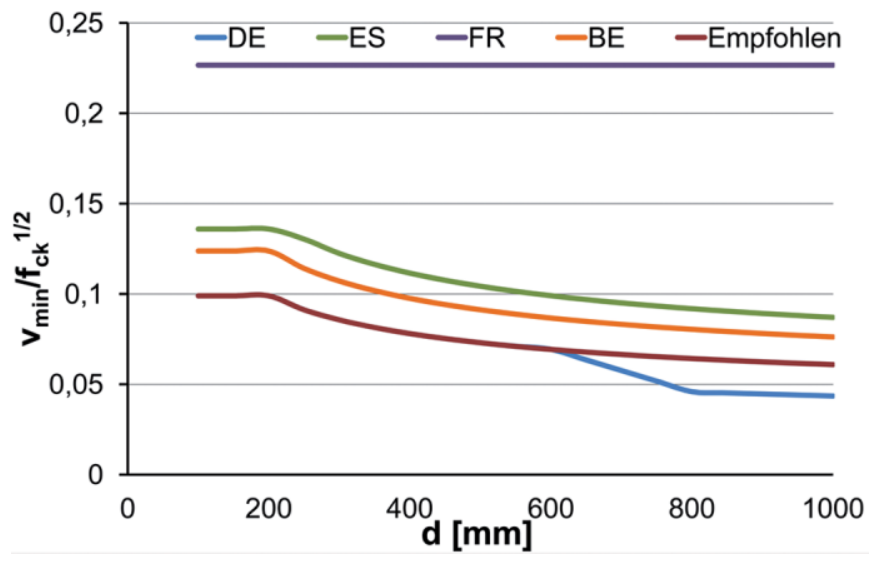

Bild 3 Vergleich der empfohlenen $v_{\min }$-Werte für Querkraft mit jenen, welche von Deutschland, Spanien, Frankreich und Belgien verwendet werden Comparison of advisory value with the chosen values of the minimum shear capacity $v_{\min }$ for the countries Germany, Spain, France and Belgium

faktor von 1,25 für die Bestimmung der Querkrafttragfähigkeit von Platten, die an den Rändern unterstützt sind. Frankreich [4] bietet zwei Ausdrücke für $v_{\min }$ an. Für Decken, welche im Lastfall „,von einem transversalen Umlagerungs-Effekt profitieren“, wurde der Ausdruck

$$
v_{\min }=\frac{0,34}{\gamma_{c}} f_{c k}^{1 / 2}
$$

festgelegt. Der transversale Umlagerungs-Effekt wurde 2007 in den „Französischen Professionellen Empfehlungen" veröffentlicht $[4,5]$. Dieser Effekt kann durch alle Anordnungen erreicht werden, welche zu einem zweidimensionalen Verhalten einer Decke beitragen:

- Durch eine solche Art der Verankerung der Längsbewehrung in der Lastverteilungszone, dass sich in Kombination mit der Bewehrung in Querrichtung weitere Lastabtragungsmechanismen einstellen können, wie z. B. fächerartige Stabwerk- oder Bogenmodelle.

- Durch eine verstärkte Querbewehrung.

- Durch eine behinderte Verformung der Decke in Querrichtung infolge der einsperrenden Wirkung ihrer unmittelbaren Umgebung (weitere angrenzende Decken, Randträger, Auflagereffekte von nahen oder entfernten Traglastelementen und/oder Windaussteifung, etc.), welche normalerweise in beinahe allen Gebäuden gefunden werden kann. Hierdurch tritt Gewölbewirkung auf. Weil sich das Gewölbe schon auf den umgebenden Beton abstützen kann, ist kein weiteres verankertes Zugband erforderlich.

Des Weiteren übernimmt Frankreich bei Decken ohne Querverteilung auch den empfohlenen Mindestwert $v_{\min }$, nämlich z.B. für Fälle ohne Gewölbewirkung (Decken mit Auskragungen bzw. freien Rändern oder größeren Öffnungen). Wenn man $v_{\min }$ gemäß Gl. (12) einführt, so wird die Grundgleichung Gl. (1) nur selten maßgebend, was in Frankreich auch tatsächlich der Fall ist. 
Das französische Argument, wonach Decken ein Verhalten zeigen, welches sich grundsätzlich von jenem der Balken unterscheidet, ist es wert, weiter betrachtet zu werden. Es ist tatsächlich fragwürdig, die Querkrafttragfähigkeit von Decken aufgrund von Versuchen an Balken ohne Querkraftbewehrung herzuleiten (die sogar in der Praxis nicht zugelassen werden, ohne mit einer Mindestbewehrung ausgestattet zu sein!). Decken haben nicht nur eine erheblich höhere Umlagerungsfähigkeit, was zu einer höheren Querkrafttragfähigkeit führt, sondern zeigen auch in Versuchen eine kleinere Streuung [6]. Es ist somit nicht unlogisch, dass der Bemessungswert der Querkrafttragfähigkeit bei Decken höher liegen kann als bei Balken.

Übrigens hat sich in der Baupraxis mittlerweile herausgestellt, dass eine genaue Herleitung des Wertes $v_{\min }$ von erheblicher Bedeutung ist. In den Niederlanden gibt es zum Beispiel viele Vollplattenbrücken mit relativ hohen Querschnitthöhen und niedrigen Bewehrungsgehalten (0,5-0,8\%). Die Anwendung des in EN 1992-1-1 empfohlenen Wertes $v_{\min }$ nach Gl. (9) würde dazu führen, dass viele von diesen Brücken verstärkt werden sollten. Die in diesem Aufsatz vorgeschlagene verbesserte Formulierung von $v_{\min }$ nach Gl. (8) ist vorteilhaft, weil sie den Einfluss der Fließgrenze des Bewehrungsstahls erkennt und somit für Bewehrungsstahl mit charakteristischen Fließgrenzen $\mathrm{f}_{\mathrm{yk}}=400 \mathrm{~N} / \mathrm{mm}^{2}$ und $\mathrm{f}_{\mathrm{yk}}=220 \mathrm{~N} / \mathrm{mm}^{2}$, wie in älteren Brücken oft verwendet, konsequenterweise günstigere Werte liefert. In den Niederlanden wurde mittlerweile der Wert $v_{\min }$ nach Gl. (8) weiterhin mit einem Plattenfaktor von 1,2 erweitert.

$\mathrm{Zu}$ der Formulierung der Querkrafttragfähigkeit von Decken nach den Gln. (1) und (2) wird noch angemerkt, dass ein wichtiger Aspekt der Querkrafttragfähigkeit nicht beachtet wurde, nämlich die Querkrafttragfähigkeit bei Decken mit auflagernahen Lasten. In EN 1992-1-1 wird in 6.2.2 (6) der Faktor $\beta=\mathrm{a}_{\mathrm{v}} / 2 \mathrm{~d}$ angegeben, mit dem der Beitrag einer Einzellast die Querkraftspannung am Auflager reduziert. Bei einer Decke stellt sich jedoch die Frage, welcher Ausbreitwinkel zutrifft und ob die Ausbreitung von der Vorder- oder der Hinterseite der Lastfläche anfängt. Mittlerweile wurden hierzu Forschungsprojekte in Delft und Aachen durchgeführt [7, 8]. Im fib Model Code 2010 wurde $\mathrm{zu}$ diesem Thema eine für Decken geeignete Formulierung vorgeschlagen.
Der letzte Parameter in EN 1992-1-1, Abschnitt 6.2.2 (Bauteile ohne Schubbewehrung), mit dem eine landesspezifische Auswahl getroffen werden kann, ist der Wert $\mathrm{k}_{1}$, welcher den Effekt einer axialen Kraft auf die Schubtragfähigkeit betrachtet. Es existiert ein nahezu allgemeiner Konsens bezüglich des empfohlenen Wertes $\mathrm{k}_{1}=0,15$ sowohl für Druck- als auch für Zugbeanspruchung. Deutschland führt für Druck einen geringeren Wert $\mathrm{k}_{1}=0,12$ ein und Norwegen erhöht den Wert für Zug auf $k_{1}=0,30$. Dies führt in beiden Fällen zu einer konservativeren Querkrafttragfähigkeit.

\subsection{Querkrafttragfähigkeit von Bauteilen mit Schubbewehrung}

Für die Bestimmung der Schubtragfähigkeit von Bauteilen mit Schubbewehrung wird ein einfaches, auf der Plastizitätstheorie basierendes Gleichgewichtsmodell verwendet, resultierend in den Gln. (3) und (4), (Bild 4). Der Grundgedanke dieses Modells ist, dass mit zunehmender Belastung auf das Element die Druckstreben mit einem ebenso zunehmend kleineren Winkel rotieren und damit ein wachsender Teil der Querkraftbewehrung aktiviert wird. Bei Abnahme des Winkels $\theta$ nimmt somit die Querkrafttragfähigkeit zu. Gleichzeitig nehmen jedoch auch die Betondruckspannungen in den Druckstreben zu. Beim Erreichen einer Betondruckspannung $v_{1} f_{c d}$ in den Druckstreben, worin $v_{1}$ der Reduktionsfaktor für die Druckfestigkeit des Stegbetons ist (darauf wird später eingegangen), wird bei nicht vorgespannten Betonträgern die maximale Schubtragfähigkeit erreicht. Aufgrund von Versuchsergebnissen stellt sich heraus, dass dieses System mit einer frei wählbaren Neigung der Druckstreben nur funktioniert, wenn der Winkel $\theta$ bestimmte Grenzwerte nicht überschreitet. Der in EN 1992-1-1 empfohlene Wert für die Beschränkung der Druckstrebenneigung $\theta$ ist

$$
1 \leq \cot \theta \leq 2,5
$$

Von den insgesamt 18 Ländern, die bei der Auswertung miteinbezogen wurden, definieren zehn Länder abweichende Grenzen für $\theta$ in deren nationalen Anhängen. Italien und Polen definieren eine obere Grenze von 2,0 für $\cot \theta$ anstatt 2,5. Norwegen und das Vereinigte Königreich fügen zu Gl. (13) die Bedingung hinzu, dass im Fall einer signifikanten Zugbeanspruchung $\left(\sigma_{t} \geq \mathrm{f}_{\mathrm{ctk}, 0,05}\right)$ die obere
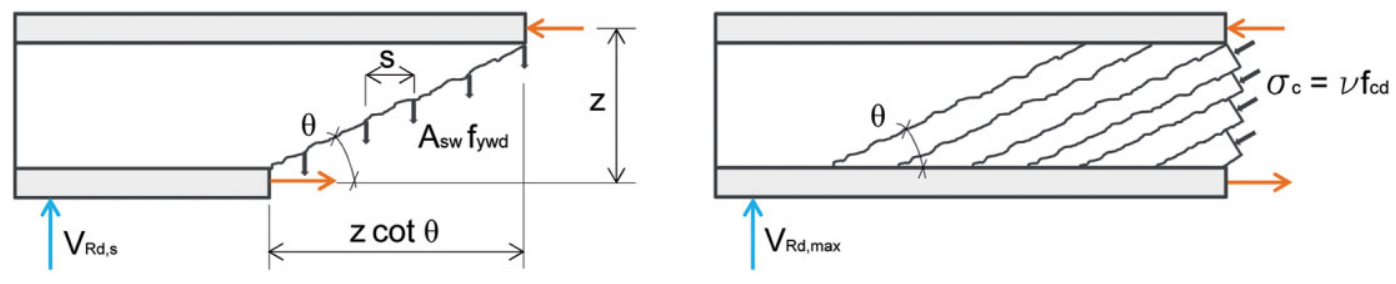

Bild 4 Basis für die Konstruktion von Bauteilen mit Querkaftbewehrung

Basis for the design of the shear reinforcement according to the variable strut inclination method 
Grenze auf $\cot \theta \leq 1,25$ beschränkt werden sollte. Österreich stellt die obere Grenze bei Zugbeanspruchung auf $\cot \theta \leq 1,67$. Frankreich schreibt für diesen Fall vor, sowohl die untere als auch die obere Grenze von $\cot \theta$ mit dem Faktor $\left(1+\sigma_{t} / f_{c t m}\right)$ zu multiplizieren. Dänemark begrenzt den Winkel $\theta$ mittels

$$
\tan \frac{\alpha}{2} \leq \cot \theta \leq 2,5
$$

wobei $\alpha$ dem Neigungswinkel der Querkraftbewehrung entspricht. Außerdem wird angegeben, dass diese Gleichung lediglich für Stahl der Klassen B und C erlaubt sei, welche eine ausreichende Umlagerungsfähigkeit besitzen. Für die Klasse des sprödesten Stahls A ist überhaupt keine Druckstrebenrotation erlaubt, also $\cot \theta=1$. Bei einer gestaffelten Bewehrung sollte weiterhin die obere Grenze von $\cot \theta$ auf 2,0 beschränkt werden. Deutschland, Ungarn und Griechenland dagegen setzen die obere Grenze für $\cot \theta$ folgendermaßen fest:

$$
1,0 \leq \cot \theta \leq \frac{1,2+1,4 \sigma_{c d} / f_{c d}}{1-V_{R d, c c} / V_{E d}} \leq 3,0
$$

wobei $\sigma_{\text {cd }}$ bei Druck mit positivem Zeichen eingeführt wird. Der Term $V_{\mathrm{Rd}, \mathrm{cc}}$ wird definiert als

$$
V_{R d, c c}=0,24 f_{c k}^{1 / 3}\left[1-1,2 \frac{\sigma_{c d}}{f_{c d}}\right] b_{w^{z}}
$$

Das heißt, dass die erlaubte untere Druckstrebenneigung $\theta$ von der relativen Größe der Querkrafttragfähigkeit $V_{\mathrm{Ed}}$ und von dem Bemessungswert der axialen Druckspannung im Querschnitt $\sigma_{\mathrm{cd}}$ abhängt. Weiterhin darf bei geneigter Querkraftbewehrung $\cot \theta$ bis 0,58 ausgenutzt werden.

Belgien geht davon aus, dass ein größerer Wert für $\cot \theta$ (also eine kleinere Neigung von $\theta$ ) auftritt, wenn das Bauteil vorgespannt ist. Es gilt

$$
1,0 \leq \cot \theta \leq \cot \theta_{\max }
$$

mit

$$
\cot \theta_{\text {max }}=\left(2+\frac{k_{1} \sigma_{c p} b_{w} d s}{A_{s w} z f_{y w d}}\right) \leq 3
$$

wobei $\sigma_{\mathrm{cp}}$ mit $\mathrm{k}_{1}=0,8$ die mittlere Druckspannung im Querschnitt ist.

Schweden unterscheidet in Bezug auf die obere Grenze von $\cot \theta$ die Fälle Stahlbeton und Spannbeton. Für Stahlbeton gilt $\cot \theta \leq 2,5$ und für Spannbeton $\cot \theta \leq 3,0$.

Eine weitere Option, welche den Ländern angeboten wird, ist, den Reduktionsfaktor $v_{1}$ für die Druckfestigkeit der Druckstreben (zur Bestimmung der maximalen Querkrafttragfähigkeit $V_{\mathrm{Rd} \text {,max }}$ nach Gl. (4)) zu wählen. Der empfohlene Wert für $v_{1}$ ist

$$
v_{1}=0,6\left[1-\frac{f_{c k}}{250}\right]
$$

Von den insgesamt 18 Ländern weichen nur drei von den empfohlenen Werten ab. In Bild 5 wird eine Übersicht mit dem empfohlenen bzw. den abweichenden Werten gegeben.

Bild 5 zeigt, dass Italien und Dänemark nur wenig von den vorgeschlagenen Werten von $v_{1}$ abweichen. Die in Deutschland angenommen Werte für $v_{1}$ sind jedoch erheblich größer als alle anderen in Europa verwendeten Werte. Ein weiterer beachtenswerter Aspekt bezüglich des Querkraftwiderstands von Bauteilen mit Schubbewehrung ist der Einfluss der Vorspannung. Diese wirkt sich zwar nicht auf die Neigung der Betondruckstreben $\cot \theta$ aus, hat aber einen Einfluss auf den maximalen Querkraftwiderstand $V_{\text {Rd,max }}$. In Gl. (4) wird dieser Tatsache durch den Faktor $\alpha_{c w}$ Rechnung getragen, welcher einen maximalen Wert von 1,25 für kleine bis mäßige axiale Vorspannung annehmen kann, Bild 6.

Von den 18 ausgewerteten Ländern haben 17 den vorgeschlagenen Wert in EN 1992-1-1 übernommen. Lediglich

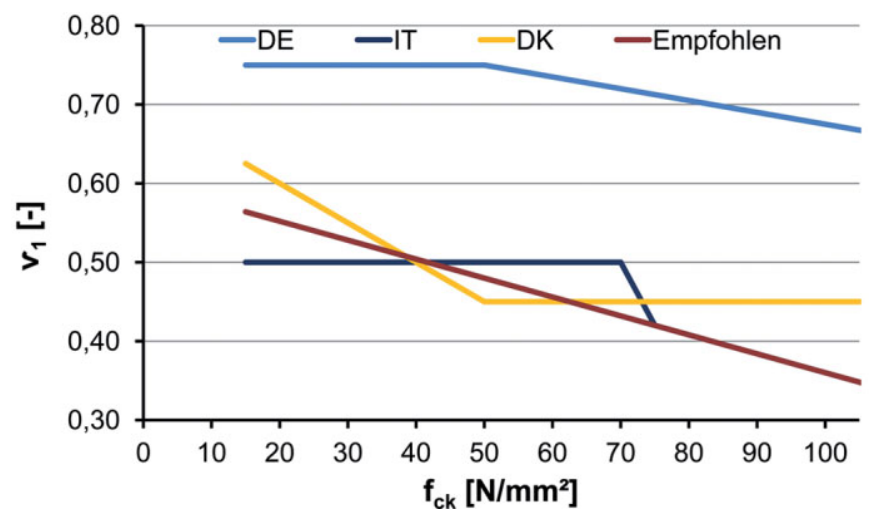

Bild 5 Vergleich der Werte für den Reduktionsfaktor $v_{1}$ für den Fall vertikaler Bügel: Empfohlene Werte und abweichende Wahl von Deutschland, Italien und Dänemark

Comparison between the values of the reduction factor $v_{1}$ for the concrete compressive strength in the struts: recommended value and value chosen by Germany, Italy and Denmark

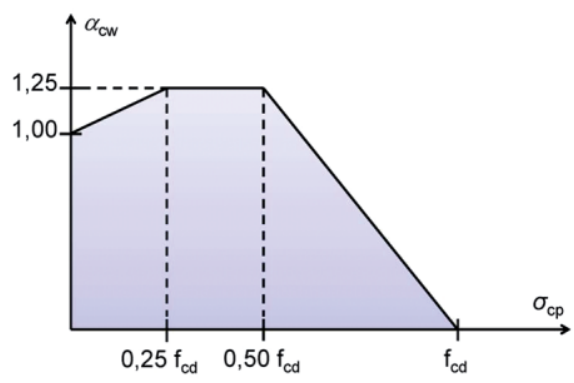

Bild 6 In EN 1992-1-1 empfohlene Werte für den Faktor $\alpha_{c w}$ zur Berücksichtigung des Einflusses der Vorspannung auf die maximale Querkrafttragfähigkeit $V_{R d, m a x}$

Advisory values for the factor $\alpha_{c w}$ to regard the influence of prestressing on the upper value of the shear capacity 
Deutschland definiert den konservativeren Wert $\alpha_{\mathrm{cw}}=1$ für alle Fälle.

Es ist deutlich, dass Deutschland in Bezug auf die Formulierung des Querkraftwiderstands erheblich von den in EN 1992-1-1 vorgeschlagenen Werten abweicht, wo die anderen Länder die vorgeschlagenen Werte weitgehend übernommen haben. Eine Überprüfung der Zusammenhänge zwischen den abweichenden Annahmen der Grenzwerte der Druckstrebenneigung, des Reduktionsfaktors $v_{1}$ und des Vorspannfaktors $\alpha_{c w}$ ist deshalb interessant. Es sollte hierbei beachtet werden, dass auch der Dauerstandsfaktor $\alpha_{c c}$ in die Tragfähigkeit der Druckstreben eingeht, weil er den Rechenwert der Betondruckfestigkeit mitbestimmt $\left(\mathrm{f}_{\mathrm{cd}}=\alpha_{\mathrm{cc}} \mathrm{f}_{\mathrm{ck}} / \gamma_{\mathrm{c}}\right)$. Bild 7 zeigt die Beziehung zwischen der berechneten Querkrafttragfähigkeit $\mathrm{V}_{\mathrm{Rd}} / \mathrm{b}_{\mathrm{w}} \mathrm{df}_{\mathrm{ck}}$ und dem Faktor $\left(\rho_{\mathrm{sw}} \cdot \mathrm{f}_{\mathrm{ywd}}\right) / \mathrm{f}_{\mathrm{ck}}$. Es wurde von einer Betonfestigkeitsklasse C30/37 und einer rechnerischen Fließspannung in der Querkraftbewehrung von $\mathrm{f}_{\mathrm{ywd}}=500 / 1,15=435 \mathrm{MPa}$ ausgegangen. Die Querkrafttragfähigkeit nach EN 1992-1-1 wurde für die Werte des Dauerstandsfaktors $\alpha_{c c}=1,0$ und 0,85 bestimmt. Hiermit decken die Linien weitgehend die nationalen Formulierungen der 18 betrachteten Länder ab. Es stellt sich heraus, dass die deutsche Formulierung gegenüber der in EN 1992-1-1 vorgeschlagenen Formulierung mit Dauerstandsfaktor $\alpha_{c c}=0,85$ mehr Querkraftbewehrung verlangt sowie auch eine erheblich höhere maximale Querkraft zulässt. Bild 8 zeigt einen ähnlichen Vergleich, wobei mit einer Betonfestigkeitsklasse C45/55 und einer rechnerischen mittleren Vorspannung $\sigma_{\mathrm{cd}}=6,5 \mathrm{MPa}$ gerechnet wird. Dies führt zum maximal möglichen Vorspannfaktor $\alpha_{\mathrm{cw}}=1,25$ (Bild 6) für die Berechnungen nach EN 1992-1-1 (in Deutschland gilt $\alpha_{\mathrm{cw}}=1,0$ unabhängig von der Vorspannung). Aus Bild 8 stellt sich heraus, dass die maximal zulässige Querkraft nach DIN EN 1992-1-1 auch für Spannbeton erheblich höher ist als die nach EN 1992-1-1, trotzdem allerdings in beiden Fällen mit dem Dauerstandsfaktor $\alpha_{c c}=0,85$ gerechnet wird. Wenn in EN 1992-1-1 von $\alpha_{c c}=1,0$ (empfohlener Wert) ausgegangen wird, so wird der Unterschied kleiner.

Eine Untersuchung von SIGRIST [9] bestätigt, dass die Formulierungen nach DIN EN 1992-1-1 signifikant von denen in EN-1992-1-1 abweichen. Des Weiteren zeigt sich jedoch auch, dass die maximale Querkrafttragfähigkeit nach DIN EN 1992-1-1 mit der neuen Formulierung nach Model Code 2010, Level II, gut übereinstimmt.

Ganz allgemein gesprochen hat die Formulierung der Querkrafttragfähigkeit nach Gln. (3) und (4) den Vorteil, dass sie die weitgehend einfachste Lösung für die Bemessung von Bauteilen mit Querkraftbewehrung bietet. Die Normalkräfte werden den Flanschen und die Querkräfte dem Steg zugewiesen. Weiterhin stellt das Modell ein transparentes Gleichgewichtssystem dar. Mittlerweile hat sich jedoch auch herausgestellt, dass diese Einfachheit auch ihre Schattenseite hat. Vor allem bei der Bestimmung der Querkrafttragfähigkeit von bestehenden Brücken kommt es darauf an, die Berechnung so wirklich-

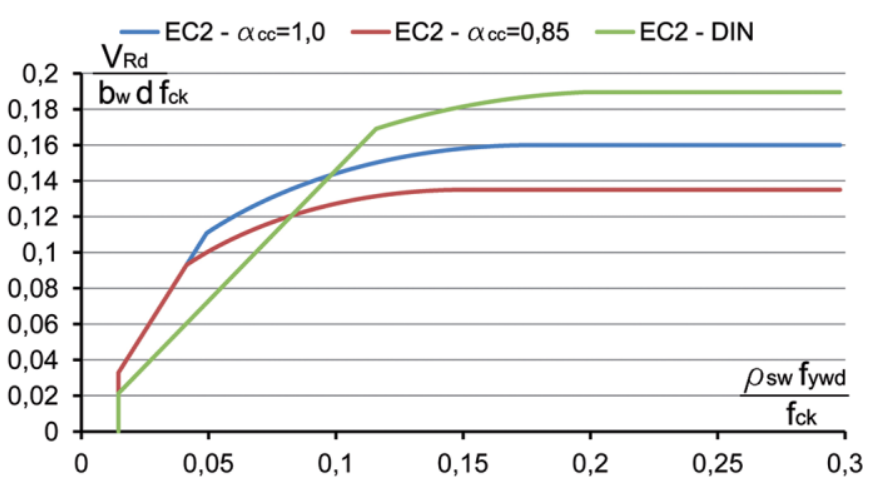

Bild 7 Querkrafttragfähigkeit von Bauteilen aus Stahlbeton nach EN 1992-1-1 (mit zwei unterschiedlichen Dauerstandswerten $\alpha_{c c}$ ) und DIN EN 1992-1-1, für eine Betonfestigkeitsklasse C30/37 Shear resistance of reinforced concrete members according to EN 1992-1-1 (with two different values of the sustained loading factor $\alpha_{c c}$ ) and DIN EN 1992-1-1 for a concrete strength class C45/55

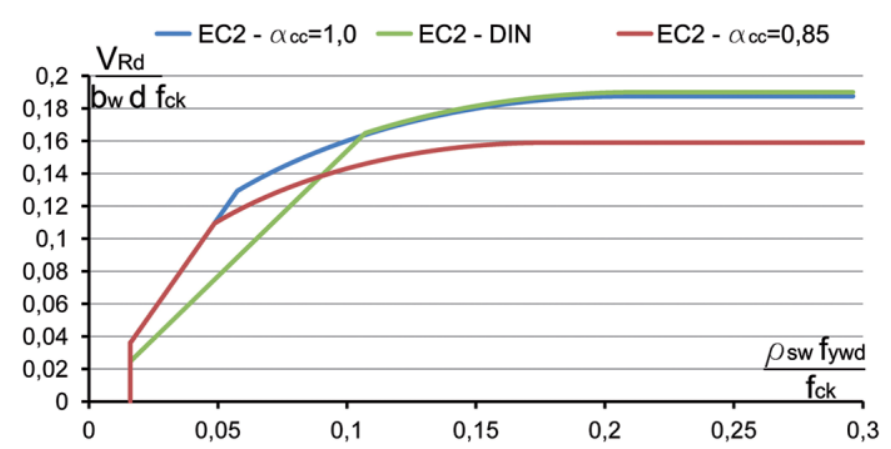

Bild 8 Querkrafttragfähigkeit von Bauteilen aus Spannbeton nach EN 1992 1-1 (mit Dauerstandsbeiwerten $\alpha_{c c}=0,85$ und 1,0 und DIN EN 1992$1-1$, für eine Betonfestigkeitsklasse $\mathrm{C} 45 / 55$ und eine rechnerische mittlere Vorspannung $\sigma_{\mathrm{cd}}=6,5 \mathrm{~N} / \mathrm{mm}^{2}$ im Querschnitt Shear resistance of prestressed concrete members according to EN 1992-1-1 (with sustained loading factors $\alpha_{c c}=0,85$ and 1,0 respectively) and DIN EN 1992-1-1 for a concrete strength class C45/55 and a design average prestress $\sigma_{\mathrm{cd}}=6,5 \mathrm{~N} / \mathrm{mm}^{2}$ in the cross section

keitsnah und zuverlässig wie möglich durchführen $\mathrm{zu}$ können. Es geht dabei oft um die Entscheidung, die Konstruktionen zu verstärken, was mit großen Investitionen einhergehen kann. In fib Model Code 2010 [10] werden deshalb Modelle zur Bestimmung des Querkraftwiderstands mit unterschiedlicher Komplexität angeboten. Die für die Bestimmung des Querkraftwiderstands meist fortgeschrittene Methode basiert auf der „Modified Compression Field Theorie“. Die Unterschiede zwischen dieser Methode und der Methode nach EN 1992-1-1 können erheblich sein, wie zum Beispiel in [11] nachgewiesen wurde.

\section{Durchstanzen}

Durchstanzen ist ein Phänomen, welches im Laufe der Jahre intensiv studiert wurde. Bemessungsgleichungen mit ihren Koeffizienten für die Berechnung des Durchstanzwiderstands von Betonflachdecken basieren auf Testresultaten und statistischen Ableitungen. In der 
EN 1992-1-1 wird die Durchstanztragfähigkeit für Decken ohne Schubbewehrung definiert als

$$
v_{E d}=\beta \frac{V_{E d}}{u_{1} d} \leq v_{R d, c}
$$

wobei

$$
v_{R d, c}=C_{R d, c} k\left(100 \rho_{l} f_{c k}\right)^{1 / 3}+k_{1} \sigma_{c p} \geq\left(v_{\min }+k_{1} \sigma_{c p}\right)
$$

Für Decken mit Durchstanzbewehrung folgt die Tragfähigkeit aus

$$
v_{R d, s}=0,75 v_{R d, c}+1,5\left(\frac{d}{s_{r}}\right) A_{s w} f_{y w d, e f}\left(\frac{1}{u_{1} d}\right) \sin \alpha
$$

Der Durchstanzwiderstand wird durch eine obere Schranke begrenzt:

$$
v_{E d}=\beta \frac{V_{E d}}{u_{0} d} \leq v_{R d, \max }
$$

wobei sich der vorgeschlagene Wert für $v_{R d, \max }$ mit

$$
v_{R d, \text { max }}=0,40 \cdot v \cdot f_{c d}
$$

ergibt. Die wichtigsten Unterschiede treten im Zusammenhang mit Gl. (23) auf. In der ehemaligen Pränorm ENV 1992-1-1 hatte die Gleichung für den maximalen Durchstanzwiderstand die Gestalt $v_{\mathrm{Rd} \text {,max }}=1,6 v_{\mathrm{Rdc}}$. Man dachte jedoch, dass es passender wäre, die obere Grenze für die Durchstanztragfähigkeit als eine Funktion der Betondruckfestigkeit $\mathrm{f}_{\mathrm{cd}} \mathrm{zu}$ definieren. Die Logik dahinter geht auf die Unterstellung zurück, dass die maximale Durchstanztragfähigkeit erreicht wird, wenn der Betonbogen an der Stelle der Verbindung mit der Stütze auf Druck versagt. Gemäß dieser Versagensart sollte der Stützendurchmesser ebenfalls als Eingangsparameter berücksichtigt werden. Der Originalausdruck für die maximale Tragfähigkeit war:

$$
v_{R d, \max }=0,50 \cdot v \cdot f_{c d}
$$

Dieser Ausdruck wurde früher im CEB-FIP Model Code for Concrete Structures 1990 vorgeschlagen. Gegen diese Formulierung entstanden jedoch Einwände aufgrund von Vergleichen mit Versuchsergebnissen, die nachwiesen, dass die Gl. (24) unkonservativ sei [12, 13]. Im Jahr 2009 wurde deshalb entschieden, die Gleichung zu ändern und durch Gl. (23) zu ersetzen. Die Reduktion des Vorfaktors von 0,5 auf 0,4 ist dabei auf numerische Untersuchungen von MANCINI [14] zurückzuführen. Die FEM-Analyse zeigte, dass der Vergrößerungsfaktor $\beta$, mit dem auf den Effekt einer exzentrischen Belastung Rücksicht genommen wird, nur angemessen funktioniert, wenn dieser nach Gl. (19) auf den kritischen Rundschnitt $\mathrm{u}_{1}$ im Abstand $2 \mathrm{~d}$ von der Lastfläche angewendet wird, jedoch nicht nach Gl. (22) auf den Rundschnitt $\mathrm{u}_{0}$ am Stützenrand. Um die damit zusammenhängende Unstimmigkeit zu kompensieren, wurde der maximale Durchstanzwider- stand in EN 1992-1-1 2009 reduziert (durch Verminderung des Vorfaktors 0,5 auf 0,4).

Im Zusammenhang mit den Werten, welche in den nationalen Anhängen enthalten sind, stellte sich heraus, dass viele Länder von Gl. (23) abweichen. In zehn Ländern wird nach wie vor am ehemaligen Ausdruck $v_{\mathrm{Rd}, \max }=$ $0,5 \vee \mathrm{f}_{\mathrm{cd}}$ festgehalten. Dies führt jedoch bereits $\mathrm{zu}$ einem Unterschied von $25 \%$ im Vergleich zum vorgeschlagenen Wert. Schweden, Norwegen, Österreich und das Vereinigte Königreich definieren alle zwei oberen Grenzen, von denen die kleinere zutrifft. So definiert Österreich

$$
V_{R d, \text { max }} \leq 0,40 v f_{c d} u_{0} d
$$

in Kombination mit

$$
V_{R d, \max } \leq \kappa v_{R d, c} u_{1} d
$$

worin $\kappa=1,40$ für $\mathrm{d} \leq 200 \mathrm{~mm}$ und $\kappa=1,60$ für $\mathrm{d} \geq$ $700 \mathrm{~mm}$ ist.

Schweden und Norwegen definieren

$$
V_{R d, \max } \leq 0,50 \cdot v \cdot f_{c d} \cdot u_{0} \cdot d
$$

in Kombination mit

$$
V_{R d, \max } \leq 1,60 v_{R d, c} u_{1} d
$$

und das Vereinigte Königreich definiert

$$
\begin{aligned}
& V_{R d, \text { max }} \leq 0,40 v f_{c d} u_{0} d \\
& v_{R d, \text { max }}=2,0 v_{R d, c}
\end{aligned}
$$

Deutschland wählt den integralen Ausdruck

$$
v_{R d, \max } \leq 1,40 v_{R d, c}
$$

Finnland akzeptiert keine der genannten Ausdrücke, weil davon ausgegangen wird, dass diese auf der unsicheren Seite liegende Ergebnisse liefern. Stattdessen erklärt man die eigene alte Norm als gültig.

In Bezug auf die Anwendung von Durchstanzbewehrung fällt weiterhin auf, dass Deutschland und Österreich die Durchstanzbewehrung nach Gl. (21) in den ersten beiden Bewehrungsreihen signifikant erhöhen.

Das folgende Beispiel illustriert die Unterschiede zwischen den diversen Fassungen in Bezug auf die maximale Durchstanztragfähigkeit. Eine Decke mit Abmessungen gemäß Bild 9 wird betrachtet. Die Betonfestigkeitsklasse C40/50 wird angenommen.

Die empfohlene verbesserte Gl. (23) ergibt den Wert $\mathrm{V}_{\mathrm{Rd} \text {, max }}=1157 \mathrm{kN}$, wo nach der alten Gl. (24) noch $1446 \mathrm{kN}$ erlaubt waren. Die deutsche Version Gl. (28) führt $\mathrm{zu}$ dem niedrigsten Wert aller Länder, nämlich 


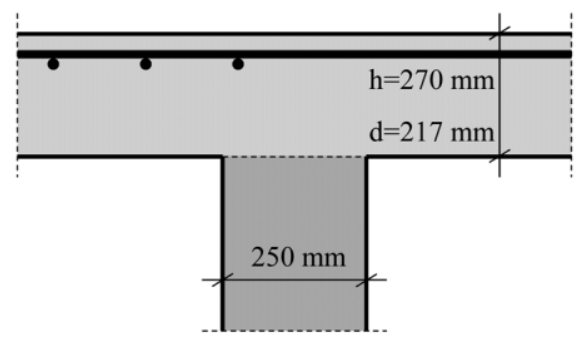

Bild 9 Beispiel für den maximalen Durchstanzwiderstand Example for the determination of the upper value of the punching shear capacity

$833 \mathrm{kN}$, was lediglich 72\% des Ergebnisses entspricht, welches man mit dem vorgeschlagenen Ausdruck Gl. (23) bekommt. Dabei ist noch zu beachten, dass der Unterschied zwischen dem Ergebnis nach dem deutschen Ausdruck Gl. (28) und jenem über den der empfohlenen Gl. (23) noch größer wird, falls die Querschnittshöhe der Decke wächst (in Folge des Maßstabseffekts enthalten in $\mathrm{V}_{\mathrm{Rd}, \mathrm{c}}$ ).

Eine Auswertung von HeGger et al. [13] hat gezeigt, dass der Multiplikationsansatz, obwohl die obere Durchstanztragfähigkeit ein über einen Vorfaktor definiertes Vielfaches der Durchstanztragfähigkeit einer Decke ohne Durchstanzbewehrung ist, z.B. Gl. (28), im Prinzip gut geeignet ist. Über diesen Vorfaktor kann auch auf die Effektivität der Durchstanzbewehrung Rücksicht genommen werden.

Anfang 2013 hat es eine neue Beratung über den einzuhaltenden Ausdruck für $\mathrm{V}_{\mathrm{Rd} \text {,max }}$ gegeben. Das hat dazu geführt, dass der Multiplikationsanzatz als Grundlage angenommen wurde und die Wahl des Vorfaktors den Ländern überlassen wird.

\section{Zusammenfassung}

1. In den Kapiteln über Querkraft und Durchstanzen werden im Eurocode 214 länderspezifische Parame-

\section{Literatur}

[1] KöNIG, G.; FISCHER, J.: Model Uncertainties of Design Equations for the Shear Capacity of Concrete Members without Shear Reinforcement. In: CEB Bulletin 224, Lausanne (1995).

[2] WALRAVEN, J. C.: Background Document for ENV 1992-1-1: 2001 Chapter 6.2 Shear. Delft University of Technology (2002).

[3] ReInecK, K. H.: Überprüfung des Mindestwerts der Querkrafttragfähigkeit in EN 1992-1-1. Projekt A3, DIBt FV ZP 52-5-7-270-1281/05, Bericht März 2007.

[4] Recommandations professionelles pour l'application de la norme NF EN 1992-1-1 et de son annexe nationale (NF P 18-711-1/NA-Eurocode 2, Partie 1-1), www.ff.batiment.fr.

[5] Chauvel, D.; Thonnier, H.; Coin, A.; Ile, N.: Shear resistance of slabs not provided with shear reinforcement. CEN/TC250/SC02N726, 2007. ter definiert. Von 18 Ländern weichen 17 in maximal vier Fällen von den empfohlenen Werten ab. Deutschland weicht in elf Fällen ab und ist damit Spitzenreiter.

2. Große Unterschiede treten in Bezug auf den minimalen Wert $v_{\text {min }}$ für die Querkrafttragfähigkeit von Bauteilen ohne Schubbewehrung auf. Die im französischen nationalen Anhang eingeführte Gleichung liefert Resultate, welche 2,5 bis 3,0 Mal höher sind als jene, welche man mit dem empfohlenem Ausdruck für $v_{\min }$ enthält. Der Ausdruck für $v_{\min }$ sollte auf alle Fälle durch Gl. (8) ersetzt werden.

3. Im Hinblick auf die Schubtragfähigkeit von Bauteilen mit Schubbewehrung definieren zehn Länder abweichende Grenzen für die zulässige Druckstrebenneigung. Dänemark berücksichtigt die Neigung der Schubbewehrung in der Definition des zulässigen Intervalls für $\theta$. Deutschland, Ungarn und Griechenland schreiben eine Berechnung für die minimale Druckstrebenneigung vor, welche abhängig von dem Bemessungswert der Normalspannung und der relativen Größe der Bemessungsquerkraft ist. Auch Belgien erkennt den Einfluss der Vorspannung auf die untere Grenze der Druckstrebenneigung.

4. Für Bauteile aus Stahlbeton und Spannbeton ist die maximale Querkrafttragfähigkeit nach DIN EN 19921-1 erheblich größer als jene nach EN 1992-1-1 in allen anderen 17 Länder, die in dem Vergleich berücksichtigt wurden.

5. Bezüglich Durchstanzen fällt die große Variation der oberen Grenze des Durchstanzwiderstands auf. Einige Länder bevorzugen eine Definition der oberen Grenze als ein Vielfaches der Tragfähigkeit einer entsprechenden Decke ohne Schubbewehrung. In der, unter den Auspizien von Eurocode Commission TC-250, neu eingestellten Arbeitsgruppe TG-4 wurde der obere Grenzwert der Durchstanztragfähigkeit mittlerweile durch den in Deutschland schon üblichen Ausdruck Gl. (23) ersetzt, wobei die Wahl des Vorfaktors den Ländern überlassen wird.

[6] YANG, Y.; DEN UiJl, J. A.; WAlRAVEN, J. C.: Shear capacity of concrete structures influenced by concrete strength variation in width direction. Report 25.5-12-07, Delft University of Technology, Dec. 2012.

[7] LANDSOGHT, E.: Shear in Reinforced Concrete Slabs under Concentrated Loads close to Supports. Dissertation, 14. June 2013.

[8] ReIsSEN, K.; Hegger, J.: Experimentelle Untersuchungen zur mitwirkenden Breite für Querkraft von einfeldrigen Fahrbahnplatten. In: Beton- und Stahlbetonbau 108 (2013), Heft 2, S. 96-103.

[9] SIGRIST, V.: Assessement of Structural Concrete Members using the fib Model Code 2010 Shear Provisions. IABSE Conference on Assessment, Upgrading and Refurbishment of Infrastructures, Rotterdam, May 6-8, 2013. 
[10] fib Model Code for Concrete Structures 2010, fib-Bulletins 65 und 66, March 2012.

[11] Huber, H.; Schweighofer, A.; Kollegger, J.: Shear strength of over 50 years old post-tensioned concrete bridge girders. fib Symposium Tel-Aviv, 22.-24. April 2013, Proceedings, S. 273-376.

[12] Fraser, A. S.; Jones, A. E. K.: Effectiveness of punching shear reinforcement to EN 1992-1-1: 2004. The Structural Engineer 87 (10), 19. Mai 2009, S. 23-27.

[13] Hegger, J.; Walraven, J. C.; Häusler, F.: Zum Durchstanzen von Flachdecken nach Eurocode 2. In: Beton- und Stahlbetonbau 105 (2010), Heft 4, S. 206-214.

[14] Mancini, G.; Bertagnoli, G.: Punching shear - What happens if the column is not symmetrically loaded? Präsentation für CEN TC 250 SC2, Turin, 11. November 2008 (unveröffentlicht).
Autoren

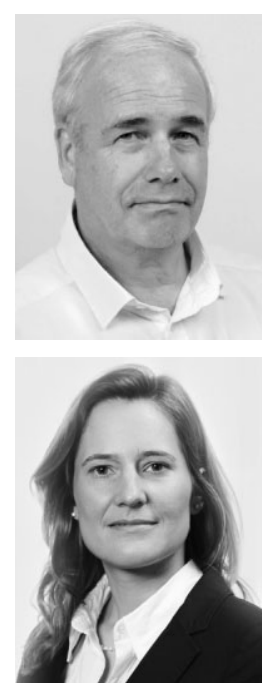

Prof. dr.ir. Dr.-Ing. E.h. Joost Walraven Delft University of Technology

Department of Design and Construction, Structural and Building Engineering Stevin II-North 2.02 2629 Delft, Netherlands j.c.walraven@tudelft.nl

Dipl.-Ing. Dr. techn. Susanne Gmainer Smart Minerals $\mathrm{GmbH}$

Reisnerstraße 53

1030 Wien

gmainer@smartminerals.at

vormals:

TU Wien, Institut für Tragkonstruktionen/ Betonbau 